\title{
Multicenter phase II clinical study of the efficiency and safety of capecitabine plus intermittent oxaliplatin with bevacizumab as first-line therapy in patients with metastatic colorectal cancer: the VOICE trial
}

Chihiro Kosugi ( $\nabla$ ckosugi0126@yahoo.co.jp )

Teikyo University Chiba Medical Center

Keiji Koda

Teikyo University Chiba Medical Center

Tadamichi Denda

Chiba Cancer Center

Keiichiro Ishibashi

Saitama Medical University

Hideyuki Ishida

Saitama Medical University

Kazuhiro Seike

Odawara Municipal Hospital

Haruhito Sakata

Saisei Hospital

Shinji Yanagisawa

Kimitsu Chuo Hospital

\section{Akinari Miyazaki}

Funabashi Municipal Medical Center

Wataru Takayama

Chiba Prefectural Sawara Hospital

Naoto Koike

Seirei Sakura Citizen Hospital

Hiroaki Shimizu

Teikyo University Chiba Medical Center

Hisahiro Matsubara

Chiba University

\section{Research Article}

Keywords: Metastatic colorectal cancer stop-and-go strategy, Reintroduction of oxaliplatin, CAPOX, Peripheral sensory neuropathy

Posted Date: April 6th, 2021

DOI: https://doi.org/10.21203/rs.3.rs-288081/v1 
License: (c) (i) This work is licensed under a Creative Commons Attribution 4.0 International License. Read Full License

Version of Record: A version of this preprint was published at International Journal of Colorectal Disease on August 9th, 2021. See the published version at https://doi.org/10.1007/s00384-021-03995-7. 


\section{Abstract}

Background: In the first-line treatment of metastatic colorectal cancer (mCRC) patients with CAPOX plus bevacizumab, the optimal duration of maintenance treatment without oxaliplatin to avoid discontinuation of therapy due to peripheral sensory neuropathy (PSN) remains unknown. The aim of this phase II study was to evaluate the efficacy and safety of combination therapy with five-cycle CAPOX (capecitabine plus oxaliplatin) plus bevacizumab, followed by five-cycle maintenance therapy with capecitabine plus bevacizumab and reintroduction of CAPOX plus bevacizumab for five cycles, with a preplanned oxaliplatin intermittent strategy in $\mathrm{mCRC}$.

Methods: Patients with untreated mCRC were administered CAPOX [oxaliplatin $130 \mathrm{mg} / \mathrm{m}^{2}$ and capecitabine (2000 $\mathrm{mg} / \mathrm{m}^{2}$ daily) as intermittent treatment for 14 days, followed by a 7-day treatment-free interval, every 3 weeks] + bevacizumab $(7.5 \mathrm{mg} / \mathrm{kg})$ every 3 weeks for five cycles, maintenance treatment without oxaliplatin for five cycles, and CAPOX + bevacizumab reintroduction for five cycles or upon tumor progression. The primary endpoint was progressionfree survival (PFS), and the secondary endpoints were the time to treatment failure (TTF), overall survival, response rate (RR), and safety.

Results: Forty-seven patients who fulfilled the inclusion criteria were enrolled in the evaluation of efficacy and safety. The relative dose intensity and the cumulative dose of oxaliplatin during the overall treatment period were $649.1 \mathrm{mg} / \mathrm{m}^{2}$ and 1132.5 mg, respectively. Median PFS was 14.1 months (95\% confidence interval [CI], 8.6-19.5), and median TTF was 12.3 months (95\% Cl, 10.3-14.3). The objective RRs were 51.1\% (24/47) during induction therapy, 58.3\% (21/36) during maintenance therapy, and $63.6 \%$ (14/22) during reintroduction therapy. The frequency of patients with neutropenia, diarrhea, PSN, venous thromboembolism, or grade $\geq 3$ allergic reactions was $2.1 \%$.

Conclusion: CAPOX plus bevacizumab therapy with a preplanned intermittent oxaliplatin strategy consisting of brief fivecycle induction therapy, five-cycle maintenance therapy with capecitabine plus bevacizumab, and five-cycle reintroduction therapy consisting of CAPOX plus bevacizumab is safe and effective for mCRC patients.

Trial registration: This trial was registered with the University Hospital Medical Information Network on 7 June 2011 (UMIN ID: 000005732).

\section{Background}

In the 1980s, the standard first-line treatment for patients with unresectable metastatic colorectal cancer (mCRC) was the 5-fluorouracil (5-FU)/leucovorin (LV) regimen [1, 2]. In the 2000s, the availability of new drugs, such as irinotecan (irinotecan + 5-FU/LV: FOLFIRI) and oxaliplatin (oxaliplatin + 5-FU/LV: FOLFOX), improved the prognosis of patients with mCRC [3-6]. The addition of the monoclonal antibody drug bevacizumab, which is effective against vascular endothelial growth factor (VEGF), to irinotecan plus bolus 5-FU (IFL) resulted in significant benefits regarding overall survival (OS) and progression-free survival (PFS) [7], and combinations of FOLFOX or FOLFIRI with monoclonal antibodies against VEGF or epidermal growth factor receptor (EGFR) and FOLFOXIRI (5-FU/LV/oxaliplatin/irinotecan) with anti-VEGF monoclonal antibodies [8] are currently recommended as first-line treatments for patients with mCRC in the National Comprehensive Cancer Center Network (NCCN) guidelines.

Kabbinavar et al. reported prolonged survival by combining bevacizumab with 5-FU/LV as first-line therapy [9], and the additive effect of bevacizumab to FOLFOX and capecitabine/oxaliplatin (CAPOX) was demonstrated in the NO16966 study [10]. CAPOX had similar efficacy to regimens based on infusional 5-FU/LV combined with oxaliplatin in some studies [10-12]. Moreover, the additive effect of bevacizumab to CAPOX improves OS or PFS $[7,10,13]$. Therefore, CAPOX with/without bevacizumab shows good efficacy [14-16] and is recommended for standard first-line chemotherapy in the NCCN guidelines. However, many patients treated with long-term oxaliplatin-based regimens develop 
peripheral sensory neuropathy (PSN) and serious hematological toxicity, leading to treatment discontinuation [17-19]. In particular, PSN is the main dose-limiting toxicity of oxaliplatin, and it negatively affects patients' daily life and causes the discontinuation of oxaliplatin before disease progression. Several recent prospective studies have suggested a stop-andgo strategy with the intermittent use of oxaliplatin in the FOLFOX regimen to avoid discontinuation of therapy due to PSN and prolong the time to treatment failure [20-23]. In the OPTIMOX1 study, Tournigand et al. [20] reported similar PFS and OS in patients with mCRC randomized to receive either FOLFOX4 until disease progression or FOLFOX7 for 6 cycles followed by maintenance without oxaliplatin for 12 cycles. Tezuka et al. [24] reported the efficiency of the oxaliplatin stopand-go concept using a regimen of mFOLFOX7 plus bevacizumab for eight cycles followed by maintenance without oxaliplatin for eight cycles in Japanese patients with unresectable mCRC.

In the CAPOX regimen, feasibility studies of intermittent oxaliplatin administration with capecitabine have been reported; however, the maintenance duration of chemotherapy combined with targeted therapy varied [25-27]. Therefore, the clinical questions of whether to reintroduce oxaliplatin in the CAPOX regimen and the optimal duration of maintenance treatment remain unknown.

To address these issues, we conducted the phase II VOICE (Bevacizumab with Oxaliplatin of Intermittent and Capecitabine therapy in the Chiba Evaluation trial in colorectal cancer) study to examine the efficacy and safety, including the incidence of PSN, of a regimen consisting of five preplanned cycles of initial CAPOX plus bevacizumab therapy, followed by five cycles of fixed-term maintenance therapy and then the reintroduction of oxaliplatin for Japanese CRC patients (UMIN ID: 000005732, 07/06/2011).

\section{Methods}

This study was a multicenter phase II clinical study to examine the efficacy and safety of intermittent oxaliplatin administration in patients with unresectable CRC receiving CAPOX plus bevacizumab as first-line therapy. The primary endpoint was progression-free survival (PFS); secondary endpoints were time to treatment failure (TTF), overall survival (OS), response rate (RR), and safety.

\section{Patient eligibility criteria}

Patients were enrolled in the study if they fulfilled the following inclusion criteria: 1) provided written informed consent; 2) CRC was definitively diagnosed histologically and was metastatic and unresectable, the disease had not received previous chemotherapy or radiation therapy, advanced CRC patients who had received no intervention expect surgical procedures, and recurrent CRC patients who had not been administered any therapy to the recurrent site; 3 ) at least one lesion was evaluable by radiological examinations within 28 days before registration; 4) age $\geq 20$ years old and provided informed consent; 5) Eastern Cooperative Oncology Group (ECOG) Performance Status (PS) of 0 or 1; and 6) vital organ function (listed below) within 14 days prior to entry: adequate hematological (white blood cell count: $>3000 / \mathrm{mm}^{3}$; neutrophil count: > 1500/ $\mathrm{mm}^{3}$; platelet count: > $10.0 \times 10^{4} / \mathrm{mm}^{3}$; hemoglobin $>9.0 \mathrm{~g} / \mathrm{dl}$ ), hepatic (total bilirubin $<1.5 \times$ the upper limit of normal (ULN); alanine aminotransferase (ALT) and aspartate aminotransferase (AST) $<2.5 \times$ ULN, or $<5$ $\times$ ULN in the case of hepatic metastases), and renal function (serum creatinine clearance $<1.5 \times$ ULN; proteinuria $<2+$ ).

Patients were excluded if there was 1) suspicion of brain metastasis or brain cancer confirmed by radiological imaging, such as head computed tomography or magnetic resonance imaging; 2) cerebrovascular disease or related symptoms within 1 year or a history of suspected complications of arterial thromboembolism, such as cerebrovascular disease or thromboembolism once within 1 year or twice; 3 ) bleeding diathesis (history of hemoptysis, including cavitation and/or necrosis in lung metastases confirmed by imaging), coagulopathy, or abnormality in coagulation factors, such as a prothrombin time-international normalized ratio $(P T-I N R) \geq 1.5 ; 4)$ history of gastrointestinal perforation within 1 year or uncontrollable peptic ulcers, uncontrollable infection, diarrhea, and hypertension; 5) symptomatic cardiovascular disease 
or symptomatic disease previously treated and history of myocardial infarction within 1 year; 6 ) women who were pregnant, possibly pregnant, wishing to become pregnant, or nursing; or 7) not appropriate for the study at the physician's assessment.

An eligibility report form was sent to the registration center at the Graduate School of Medicine, Department of Frontier Surgery, Chiba University (Chiba, Japan). Information regarding the necessary follow-up tests was then sent to the investigator from the registration center. The registration period was from February 2011 to January 2014, and the ongoing follow-up period was 2 years from enrollment of the last subject.

\section{Treatment}

Induction therapy

The protocol treatment was started within 14 days after enrollment for eligible patients. Patients received five cycles of CAPOX plus bevacizumab therapy. Intravenous oxaliplatin $\left(130 \mathrm{mg} / \mathrm{m}^{2}\right)$ was administered over $120 \mathrm{~min}$, bevacizumab $(7.5 \mathrm{mg} / \mathrm{kg}$ ) was administered by a 30-min intravenous infusion on day 1 of the 3-week cycle, and oral capecitabine (2000 $\mathrm{mg} / \mathrm{m}^{2}$ ) was administered daily on days $1-14$ every 3 weeks. This regimen constituted cycles $1-5$.

\section{Maintenance therapy}

Five cycles (cycles 6-10) were given without oxaliplatin. Capecitabine (2000 mg/m² daily on days $1-14$ every 3 weeks) plus bevacizumab $(7.5 \mathrm{mg} / \mathrm{kg}$ on day 1 of the 3-week cycle) therapy was initiated for patients with disease control (complete response (CR), partial response (PR), and stable disease (SD)) after the induction therapy.

\section{Reintroduction therapy}

Reintroduction of oxaliplatin was scheduled after 10 cycles of induction and maintenance therapy. Oxaliplatin was then reintroduced for five cycles, so that a total of 15 cycles was administered as the protocol treatment. Protocol treatment was continued as long as it did not conflict with the criteria for discontinuing treatment. However, the following defined the end of the protocol treatment: (1) The 15 cycles (induction therapy followed by maintenance therapy and then reintroduction therapy) prescribed in the protocol were completed. However, if progressive disease (PD) was confirmed before completing five cycles in the maintenance therapy, the end of protocol treatment was defined as the end of oxaliplatin reintroduction therapy. (2) The patient became operable as a result of treatment efficacy (with the day of surgery defined as the end of protocol treatment).

\section{Dose reduction and withdrawal criteria}

The types and severities of adverse events (AEs) were evaluated according to the National Cancer Institute Common Terminology Criteria for Adverse Events (NCI-CTCAE) version 4.0 [28], and PSN was assessed in accordance with the Neurotoxicity Criteria of Debiopharm (DEB-NTC) [29-31]. Dose reduction for subsequent courses was performed according to AEs that occurred during the previous course. The dose could be reduced twice, after which protocol treatment was discontinued if a third dose reduction was required. The dose could not be increased again after being reduced.

Patients were observed carefully for previously reported typical adverse drug reactions to bevacizumab and oxaliplatin, including hemorrhage, thrombosis, gastrointestinal perforation, increased blood pressure, hemotoxicity, and PSN. The dose of capecitabine was reduced for grade $\geq 3$ neutropenia, thrombocytopenia, diarrhea, hand-foot syndrome, stomatitis, skin toxicity, or other nonhematologic toxicities or for a second or subsequent occurrence of hand-foot syndrome. If PSN occurred, dose reduction or treatment withdrawal was performed. 
If grade $\geq 3$ hematological AEs (neutropenia, thrombocytopenia, other hematological toxicities) occurred, the doses of oxaliplatin and capecitabine were reduced as follows: oxaliplatin was reduced to $100 \mathrm{mg} / \mathrm{m}^{2}$, and capecitabine was reduced to $1500 \mathrm{mg} / \mathrm{m}^{2}$. If a similar AE occurred subsequently, the doses were reduced further to $85 \mathrm{mg} / \mathrm{m}^{2}$ and 1000 $\mathrm{mg} / \mathrm{m}^{2}$, respectively. If PSN occurred, dose reduction or treatment withdrawal was performed.

\section{Criteria for starting the next cycle and discontinuation criteria}

The clinical findings and laboratory test values (listed below) were evaluated in all patients on the first planned day (Day 1) of each treatment cycle. If all of the following criteria were not fulfilled, treatment was postponed until the criteria were met: 1 ) adequate hematological indices: white blood cell count $>3000 / \mathrm{mm}^{3}$ and $<12,000 / \mathrm{mm}^{3}$; neutrophil count $>$ $1500 / \mathrm{mm}^{3}$; platelet count $>75,000 \mathrm{~mm}^{3}$; hemoglobin > $9.0 \mathrm{~g} / \mathrm{dL} ; 2$ ) hepatic indexes: total bilirubin $<1.5 \times$ ULN; ALT and AST $<2.5 \times$ ULN, or $<5 \times$ ULN in the case of hepatic metastases; 3 ) renal function: serum creatinine below the institutional ULN; and 4) PSN: grade $\leq 2$.

The protocol treatment was discontinued if any of the following occurred: the development of any AEs (defined as grade $\geq 2$ hypersensitivity); the criteria for starting the next cycle were not met within 4 weeks ( 28 days) after the planned day of initiating treatment because of an $\mathrm{AE}$; further toxicity meeting the dose reduction criteria after two dose reductions; discontinuation considered necessary by the investigator because of an $\mathrm{AE}$ (even if the criteria were not met); the patient requested to discontinue treatment; death during protocol treatment; or a protocol violation was found after enrollment, making the patient ineligible [32].

\section{Statistical determination of target sample size}

In the N016966 study [10], a phase III clinical study of FOLFOX4 or CAPOX with/without bevacizumab, the median PFS by on-treatment analysis, excluding PD cases from at least 29 days after the final administration of the study drug, was 10.4 months. In the MACRO trial [25], a phase III clinical study conducted to confirm first-line CAPOX plus bevacizumab followed by CAPOX plus bevacizumab or single-agent bevacizumab as maintenance therapy, the median PFS for CAPOX plus bevacizumab was 10.4 months, and that for single-agent bevacizumab was 9.7 months. Therefore, the threshold PFS in this study was assumed to be 7 months considering PFS in the phase III study of FOLFOX4 with bevacizumab [10] and CAPOX with bevacizumab [25], and the anticipated PFS was set at 10.5 months. The level of significance was 0.05 at a power of $80 \%$, and the required number of cases determined using the Southwest Oncology Group's one-arm survival design (http://www.swogstat.org/statoolsout.html) was 42 patients. The target number of cases in this study was 50 patients, considering a dropout rate of $10 \%$.

\section{Statistical analysis}

PFS (as the primary objective of this study) and TTF (as the secondary endpoint) were estimated using the Kaplan-Meier method. The median PFS and TTF were calculated, and their two-sided 95\% confidence interval (Cl) was determined. Regarding the RR, the antitumor effect was calculated, and the exact $\mathrm{Cl}$ was used for the interval estimation. Statistical analyses were performed using SPSS, version 23 (IBM Corp., Armonk, NY, USA). Statistical significance was established at the $P<0.05$ level.

\section{Results}

\section{Patient characteristics}

From March 2011 to August 2013, fifty-five patients from 13 institutions in Japan were enrolled in this study. After excluding 8 of the 55 patients because of protocol ineligibility, the efficacy and safety analyses were conducted with 47 patients. The data cutoff was May 2017, and the median duration of follow-up at the time of analysis was 25.1 months. 
The baseline patient characteristics are summarized in Table 1. The median age was 67 years (range: $43-83$ years), 26 were colon cancer patients, and 21 were rectal cancer patients. Thirty-five (74.5\%) patients had undergone previous surgery with curative intent and were offered our treatment protocol after documentation of unresectable locallyadvanced or metastatic disease. Eleven patients (23.4\%) had previously received adjuvant chemotherapy: tegafur-uracil (UFT) and LV: three patients; UFT: one patient: S-1: three patients; mFOLFOX6: two patients; CAPOX: one patient; and capecitabine: one patient (Table 1).

\section{Treatment status}

The study flowchart of the protocol treatment is shown in Figure 1. Of the 55 enrolled patients, the protocol treatment was administered to 47 patients. The median number of cycles was 10 (range: 1-15 cycles), and the completion rate of induction therapy was $76.6 \%$ (36/47). The reason for discontinuing treatment during induction therapy in 11 patients was PD in 4 cases, AEs in 3 cases, withdrawal of consent in 1 case, and other reasons in 3 cases (cholangitis: 1 case, small bowel obstruction: 1 case, and dementia: 1 case).

The maintenance therapy with capecitabine plus bevacizumab was administered to 36 patients (76.6\%). The rate of completion of maintenance therapy was $61.1 \%$ (22/36). The reason for discontinuing treatment during maintenance therapy in 14 patients was PD in 5 cases, AEs in 4 cases, hepatectomy in 3 cases, and physician's decision in 2 cases. Five patients with confirmed PD were not moved to reintroduction therapy as stipulated in the protocol according to the physician's judgement and were shifted to second-line chemotherapy or radiotherapy.

The reintroduction therapy with CAPOX plus bevacizumab was administered to 22 patients (46.8\%). The rate of completion of reintroduction therapy treatment was $90.9 \%(20 / 22)$. The reason for discontinuing treatment during reintroduction therapy in two patients was $\mathrm{PD}$ in one case and $\mathrm{AE}$ in one case. The protocol completion rate was $42.6 \%$ (20/47). The median TTF was 12.3 months (95\% Cl: 10.3-14.3) (Fig. 2).

The total relative dose intensity was $649.1 \mathrm{mg} / \mathrm{m}^{2}$, and the cumulative dose of oxaliplatin during the overall treatment period was $1132.5 \mathrm{mg}$ (94.0\%) (Table 2). The relative dose intensities of the induction and reintroduction of CAPOX plus bevacizumab therapy were $632.0 \mathrm{mg} / \mathrm{m}^{2}$ and $518.9 \mathrm{mg} / \mathrm{m}^{2}$, respectively, and the cumulative dose of oxaliplatin after induction and reintroduction therapy was $950.0 \mathrm{mg}(92.3 \%)$ and $805.0 \mathrm{mg}$ (80.7\%), respectively.

\section{Efficacy}

The median PFS, the primary endpoint, was 14.1 months (95\% Cl: 8.6-19.5) (Fig. 3). As the secondary endpoint, the RR was $51.1 \%$ (24/47) during induction therapy, 58.3\% (21/36) during maintenance therapy, and 63.6\% (14/22) during reintroduction therapy in the 47 patients who were evaluable by the response evaluation criteria in solid tumors (RECIST) criteria (Table 3). The disease control rate was 87.2\% (41/47), 83.3\% (30/36), and 95.5\% (21/22) with induction, maintenance, and reintroduction therapy, respectively. Exploratory analysis showed that the median 0S was 27.2 months (95\% Cl: 18.9-35.5).

\section{Safety profile}

The incidence of treatment-related AEs is presented in Table 4. The rates of AEs for any grade of hematologic toxicities in the 47 patients in the safety analysis set during induction, maintenance, and reintroduction therapy, respectively, were neutropenia $(14.9 \%, 13.9 \%$, and $9.1 \%)$ and anemia $(10.6 \%, 5.6 \%$, and $0 \%)$, and the non-hematologic toxicities and their rates were general fatigue $(31.9 \%, 25.0 \%$, and $31.8 \%)$, stomatitis $(19.1 \%, 16.7 \%$, and $18.2 \%)$, nausea/vomiting (19.1\%, $11.1 \%$, and $18.2 \%)$, appetite loss $(38.3 \%, 13.9 \%$, and $9.1 \%)$, diarrhea $(21.3 \%, 5.6 \%$, and $9.1 \%)$, PSN $(55.3 \%, 44.4 \%$, and $59.1 \%)$, and hand-foot syndrome (38.3\%, 50.0\%, and 54.5\%). The AEs and rates during induction, maintenance, and reintroduction therapy, respectively, for bevacizumab-related toxicities were hypertension $(17.0 \%, 16.7 \%$, and $22.7 \%)$ and 
proteinuria $(17.0 \%, 16.7 \%$, and $22.7 \%)$. The main adverse drug reactions of grade $\geq 3$ were neutropenia, appetite loss, diarrhea, PSN, venous thromboembolism, and allergic reaction, each in one patient (2.1\% in total), and hypertension in two patients. Discontinuation of protocol treatment due to oxaliplatin-related AEs occurred in three cases by PSN (6.4\%) and three cases by allergic reactions (6.4\%). No patient experienced grade $\geq 3$ bevacizumab-related AEs, gastrointestinal perforation, clinically significant hemorrhage, or proteinuria.

\section{Discussion}

The present phase II study was conducted to evaluate the efficacy and safety of a planned stop-and-go strategy with a regimen consisting of CAPOX plus bevacizumab, followed by five cycles of fixed-term maintenance therapy and then the reintroduction of oxaliplatin as the first-line therapy in Japanese patients with unresectable mCRC.

Oxaliplatin-based chemotherapy plus bevacizumab is recommended in the guidelines of various countries as the first-line therapy for unresectable mCRC. Comparing the therapeutic effects of different oxaliplatin-based chemotherapy plus bevacizumab phase III studies with PFS as the primary endpoint, PFS in the N016966 study was 9.4 months in the FOLFOX4 plus bevacizumab group and 9.3 months in the capecitabine plus oxaliplatin (XELOX) plus bevacizumab group [10], and in the HORIZON III study, PFS in the mFOLFOX6 plus bevacizumab group was 10.3 months [33]. In the CONcePT trial [34], PFS in the intermittent administration group receiving FOLFOX7 plus bevacizumab \pm prophylactic calcium/magnesium salts, which preceded the study, was 12.0 months, and in the CRAFT trial [24], PFS in the intermittent administration group receiving FOLFOX7 plus bevacizumab was 11.8 months. Therefore, several trials using intermittent administration of CAPOX plus bevacizumab have been reported in Western countries. In the CAIRO 3 trial [26], a large randomized trial evaluating induction treatment with CAPOX plus bevacizumab and maintenance treatment with capecitabine plus bevacizumab versus only observation followed by the reintroduction of oxaliplatin, the first PFS after maintenance treatment until PD (PFS1) was 8.5 months, and the second PFS after the reintroduction of CAPOX plus bevacizumab (PFS2) was 11.7 months. In the study, a statistically significant difference was found in the median PFS2 (8.5 months in the observation group and 11.7 months in the maintenance group; hazard ratio (HR): 0.63; 95\% Cl: 0.53$0.77, \mathrm{P}<0.0001)$. In contrast, in the MACRO trial [25], which evaluated maintenance therapy with only bevacizumab following induction with CAPOX plus bevacizumab versus continuous CAPOX plus bevacizumab, no statistically significant differences were found regarding median PFS (10.4 months with CAPOX plus bevacizumab versus 9.7 months with only bevacizumab). These results showed that maintenance treatment with capecitabine plus bevacizumab was effective for patients with $\mathrm{MCRC}$.

In Japan, in the CCOG-0902 trial [27], which evaluated maintenance therapy with eight cycles of capecitabine plus bevacizumab following induction with four cycles of CAPOX plus bevacizumab and reintroduction CAPOX plus bevacizumab, PFS was 12.4 months. This result showed that CAPOX plus bevacizumab with intermittent oxaliplatin was a valid chemotherapy regimen for Japanese patients with mCRC.

In the present study, the median PFS, as the primary endpoint, was 14.1 months, which was considered acceptable according to our hypothesis, assuming a threshold and expected PFS of 7 months and 10.5 months, respectively. Our results were comparable to the CAIRO 3 trial (11.7 months) [26], MACRO (9.7 months) [25], and CCOG-0902 trial [27] (12.4 months). The design of our treatment protocol involved maintenance therapy with five cycles of capecitabine plus bevacizumab following induction with five cycles of CAPOX plus bevacizumab and reintroduction of five cycles of CAPOX plus bevacizumab. We consider that the oxaliplatin reintroduction rate of $46.8 \%(22 / 47)$ for the entire cohort, the fact that $61.1 \%(22 / 36)$ of patients who received maintenance therapy tolerated reintroduction, and the high objective RR (63.3\%) and disease control rate (95.5\%) during the reintroduction phase indicated relatively good PFS. These results suggest that our treatment plan consisting of five cycles of maintenance therapy with capecitabine plus bevacizumab and five cycles 
of reintroduction therapy with CAPOX plus bevacizumab was as feasible as an intermittent CAPOX plus bevacizumab treatment strategy.

Preplanned oxaliplatin withdrawal is a treatment strategy against PSN caused by cumulative oxaliplatin toxicity. In the present study, grade 3 or 4 PSN was observed in only $2.1 \%$ of the patients throughout the treatment period. This result was comparable to results in previous studies [25-27] and might have contributed to the longer TTF of 12.3 months. However, the timing for reintroducing oxaliplatin must be discussed. In representative studies of the stop-and-go strategy with CAPOX plus bevacizumab, oxaliplatin was stopped 18 weeks after starting treatment and maintenance therapy with capecitabine plus bevacizumab or bevacizumab alone until disease progression in the CAIRO3 trial (grade $\geq 3$ PSN rate: 10\%) [26], Turkish Oncology Group trial (grade $\geq 3$ PSN rate: 8\%) [35], and MACRO trial (grade $\geq 3$ PSN rate: 1.6\%) [25]. In the CCOG-0902 trial [27], oxaliplatin was stopped 12 weeks after starting treatment, and the regimen consisted of 24 weeks of maintenance therapy with capecitabine plus bevacizumab and reintroduction therapy with CAPOX plus bevacizumab until disease progression. In the MACRO [25] and Turkish Oncology Group trials [35] investigating 18 weeks of induction therapy with CAPOX plus bevacizumab, the relative dose intensities of oxaliplatin were $770.2 \mathrm{mg} / \mathrm{m}^{2} \mathrm{and}$ $766 \mathrm{mg} / \mathrm{m}^{2}$, respectively. In the CCOG-0902 trial [27], the cumulative dose of oxaliplatin during the overall treatment period was $1052 \mathrm{mg}$. In the present study, the total relative dose intensity was $649.1 \mathrm{mg} / \mathrm{m}^{2}$, and the total cumulative dose of oxaliplatin during the overall treatment period was $1132.5 \mathrm{mg}$. In particular, the relative dose intensities of the induction and reintroduction of CAPOX plus bevacizumab therapy were $632.0 \mathrm{mg} / \mathrm{m}^{2}$ and $518.9 \mathrm{mg} / \mathrm{m}^{2}$, respectively, and the cumulative doses of oxaliplatin during induction and reintroduction therapy were $950.0 \mathrm{mg}$ (92.3\%) and $805.0 \mathrm{mg}$ (80.7\%), respectively. The total relative dose intensity in the present study was less than that in the MACRO [25] and Turkish Oncology Group trials [35]; however, the total cumulative dose intensity of oxaliplatin and the cumulative dose of oxaliplatin during induction and reintroduction therapy in the present study were higher. We consider that the higher cumulative dose of oxaliplatin with fixed-term five-cycle maintenance therapy and five-cycle reintroduction therapy as a stop-and-go strategy prevented oxaliplatin-induced PSN and achieved good PFS and TTF in the present study.

This study has several limitations. First, the single-arm design and relatively small sample size in this study necessitate confirming our results in a larger cohort study. Second, the sizes of the target tumors were not evaluated by central review. However, the results of this study suggest that our treatment plan could be feasible as an intermittent oxaliplatin treatment strategy for Japanese patients with $\mathrm{MCRC}$. Randomized controlled trials with larger numbers of patients are recommended in Japan to confirm the efficacy and safety of this intermittent oxaliplatin strategy, as in the CAIRO3 trial [26].

\section{Conclusions}

The preplanned intermittent oxaliplatin strategy with brief five-cycle induction therapy consisting of CAPOX plus bevacizumab, five-cycle maintenance therapy consisting of capecitabine plus bevacizumab, and five-cycle reintroduction therapy consisting of CAPOX plus bevacizumab is safe and effective for Japanese MCRC patients. A higher cumulative dose of oxaliplatin prevented oxaliplatin-induced PSN, and good PFS and TTF were achieved by our fixed-term five-cycle maintenance therapy and five-cycle reintroduction therapy as a stop-and-go strategy.

\section{Declarations}

\section{Ethical approval and consent to participate}

The protocol and informed consent forms were approved by the ethics committee of Teikyo University, Tokyo, Japan (reference number: 12-089). All procedures performed in studies involving human participants were in accordance with the ethical standards of the institutional and/or research committees and with the 1964 Helsinki declaration and its later 
amendments or comparable ethical standards. The scientific and ethical validity of this study was reviewed by the Ethical Review Board of each participating facility, and the study was conducted after receiving their approval. Written informed consent was obtained from all individual participants included in the study. This study was registered with the University Hospital Medical Information Network Clinical Trials Registry (UMIN-CTR) (number: UMIN000005732).

\section{Consent for publication}

Not applicable.

\section{Availability of data and materials}

The datasets used and/or analyzed during the current study are available from the corresponding author on reasonable request.

\section{Competing interests}

The authors declare that they have no competing interests.

\section{Funding}

Not applicable.

\section{Authors' contributions}

All authors contributed to the study conception and design. CK, KK, TD, and HM: writing the protocol, data collection and interpretation, statistical analysis, and writing the paper. KI, HI, KS, HS, SY, AM, WT, NK, and HS: data collection and interpretation and writing paper. The first draft of the manuscript was written by CK, and all authors commented on previous versions of the manuscript. All authors read and approved the final manuscript.

\section{Acknowledgments}

We thank Aki Komatsu (secretary at the SOAC Data Center at the Department of Frontier Surgery, Graduate School of Medicine, Chiba University) and Tomoko Nakabayashi (secretary at the Department of Surgery, Teikyo University Chiba Medical Center) for their data collection support, and all of the patients, their families, and medical staff. We thank Jane Charbonneau, DVM, and Melissa Crawford, PhD, from Edanz Group (https://en-author-services.edanz.com/ac) for editing a draft of this manuscript.

\section{Abbreviations}

CAPOX, capecitabine/oxaliplatin; CRC, colorectal cancer; mCRC, metastatic CRC; ECOG PS, Eastern Cooperative Oncology Group Performance Status; PFS, progression-free survival; XELOX, capecitabine plus oxaliplatin; PFS1, first PFS after maintenance treatment until PD; PFS2, second PFS after reintroduction of CAPOX plus bevacizumab; HR, hazard ratio; OS, overall survival; TTF, time to treatment failure; RR, response rate; $\mathrm{Cl}$, confidence interval; PSN, peripheral sensory neuropathy; 5-FU, 5-fluorouracil; LV, leucovorin; FOLFOX, oxaliplatin in combination with 5-fluorouracil; FOLFIRI, irinotecan + 5-FU/LV; FOLFOXIRI, 5-FU/LV/oxaliplatin/irinotecan; VEGF, vascular endothelial growth factor; IFL, irinotecan plus bolus 5-FU; EGFR, epidermal growth factor receptor; NCCN, National Comprehensive Cancer Center Network; VOICE, Bevacizumab with Oxaliplatin of Intermittent and Capecitabine therapy in the Chiba Evaluation; ULN, upper limit of normal; ALT, alanine aminotransferase; AST, aspartate aminotransferase; CT, computed tomography; MRI, magnetic resonance imaging; PT-INR, prothrombin time-international normalized ratio; UMIN-CTR, University Hospital Medical Information Network Clinical Trials Registry; PD, progressive disease; AE, adverse event; NCI-CTCAE, National Cancer 
Institute Common Terminology Criteria for Adverse Events; DEB-NTC, Neurotoxicity Criteria of Debiopharm; PPS, perprotocol set; UFT, tegafur-uracil; RECIST, response evaluation criteria in solid tumors; SD, stable disease; ORR, objective response rate; $\mathrm{NE}$, not examined; $\mathrm{PR}$ partial response; $\mathrm{CR}$, complete response; $\mathrm{DCR}$, disease control rate

\section{References}

1. Petrelli N, Herrera L, Rustum Y, Burke P, Creaven P, Stulc J, et al. A prospective randomized trial of 5-fluorouracil versus 5-fluorouracil and high-dose leucovorin versus 5-fluorouracil and methotrexate in previously untreated patients with advanced colorectal carcinoma. J Clin Oncol. 1987;5:1559-65.

2. de Gramont A, Bosset JF, Milan C, Rougier P, Bouche O, Etienne PL, et al. Randomized trial comparing monthly lowdose leucovorin and fluorouracil bolus with bimonthly high-dose leucovorin and fluorouracil bolus plus continuous infusion for advanced colorectal cancer: a French intergroup study. J Clin Oncol. 1997;15:808-15.

3. de Gramont A, Figer A, Seymour M, Homerin M, Hmissi A, Cassidy J, et al. Leucovorin and fluorouracil with or without oxaliplatin as first-line treatment in advanced colorectal cancer. J Clin Oncol. 2000;18:2938-47.

4. Cheeseman SL, Joel SP, Chester JD, Wilson G, Dent JT, Richards FJ, et al. A 'modified de Gramont' regimen of fluorouracil, alone and with oxaliplatin, for advanced colorectal cancer. Br J Cancer. 2002;87:393-9.

5. Goldberg RM, Sargent DJ, Morton RF, Fuchs CS, Ramanathan RK, Williamson SK, et al. A randomized controlled trial of fluorouracil plus leucovorin, irinotecan, and oxaliplatin combinations in patients with previously untreated metastatic colorectal cancer. J Clin Oncol. 2004;22:23-30.

6. Douillard JY, Cunningham D, Roth AD, Navarro M, James RD, Karasek P, et al. Irinotecan combined with fluorouracil compared with fluorouracil alone as first-line treatment for metastatic colorectal cancer: a multicentre randomised trial. Lancet. 2000;355:1041-7.

7. Hurwitz H, Fehrenbacher L, Novotny W, Cartwright T, Hainsworth J, Heim W, et al. Bevacizumab plus irinotecan, fluorouracil, and leucovorin for metastatic colorectal cancer. N Engl J Med. 2004;350:2335-42.

8. Cremolini C, Loupakis F, Antoniotti C, Lupi C, Sensi E, Lonardi S, et al. FOLFOXIRI plus bevacizumab versus FOLFIRI plus bevacizumab as first-line treatment of patients with metastatic colorectal cancer: updated overall survival and molecular subgroup analyses of the open-label, phase 3 TRIBE study. Lancet Oncol. 2015;16:1306-15.

9. Kabbinavar FF, Hambleton J, Mass RD, Hurwitz HI, Bergsland E, Sarkar S. Combined analysis of efficacy: the addition of bevacizumab to fluorouracil/leucovorin improves survival for patients with metastatic colorectal cancer. J Clin Oncol. 2005;23:3706-12.

10. Saltz LB, Clarke S, Diaz-Rubio E, Scheithauer W, Figer A, Wong R, et al. Bevacizumab in combination with oxaliplatinbased chemotherapy as first-line therapy in metastatic colorectal cancer: a randomized phase III study. J Clin Oncol. 2008;26:2013-9.

11. Martoni AA, Pinto C, Di Fabio F, Lelli G, Llimpe FLR, Gentile AL, et al. Capecitabine plus oxaliplatin (xelox) versus protracted 5-fluorouracil venous infusion plus oxaliplatin (pvifox) as first-line treatment in advanced colorectal cancer: a GOAM phase II randomized study (FOCA trial). Eur J Cancer. 2006;42:3161-8.

12. Cassidy J, Clarke S, Díaz-Rubio E, Scheithauer W, Figer A, Wong R, et al. Randomized phase III study of capecitabine plus oxaliplatin compared with fluorouracil/folinic acid plus oxaliplatin as first-line therapy for metastatic colorectal cancer. J Clin Oncol. 2008;26:2006-12.

13. Hochster HS, Hart LL, Ramanathan RK, Childs BH, Hainsworth JD, Cohn AL, et al. Safety and efficacy of oxaliplatin and fluoropyrimidine regimens with or without bevacizumab as first-line treatment of metastatic colorectal cancer: results of the TREE study. J Clin Oncol. 2008;26:3523-9.

14. National Comprehensive Cancer Network. NCCN Guidelines for Colon Cancer. https://www.nccn.org/professionals/physician_gls/default.aspx\#colon. Accessed 06 June 2011.

Page $11 / 20$ 
15. Schmoll HJ, Van Cutsem E, Stein A, Valentini V, Glimelius B, Haustermans K, et al. ESMO Consensus Guidelines for management of patients with colon and rectal cancer. A personalized approach to clinical decision making. Ann Oncol. 2012;23:2479-516.

16. Watanabe T, Itabashi M, Shimada Y, Tanaka S, Ito Y, Ajioka Y, et al. Japanese Society for Cancer of the Colon and Rectum (JSCCR) guidelines 2010 for the treatment of colorectal cancer. Int J Clin Oncol. 2012;17:1-29.

17. Goldberg RM, Sargent DJ, Morton RF, Fuchs CS, Ramanathan RK, Williamson SK, et al. Randomized controlled trial of reduced-dose bolus fluorouracil plus leucovorin and irinotecan or infused fluorouracil plus leucovorin and oxaliplatin in patients with previously untreated metastatic colorectal cancer: a North American Intergroup Trial. J Clin Oncol. 2006;24:3347-53.

18. Argyriou AA, Polychronopoulos P, Iconomou G, Chroni E, Kalofonos HP. A review on oxaliplatin-induced peripheral nerve damage. Cancer Treat Rev. 2008;34:368-77.

19. Argyriou AA, Velasco R, Briani C, Cavaletti G, Bruna J, Alberti P, et al. Peripheral neurotoxicity of oxaliplatin in combination with 5-fluorouracil (FOLFOX) or capecitabine (XELOX): a prospective evaluation of 150 colorectal cancer patients. Ann Oncol. 2012;23:3116-22.

20. Tournigand C, Cervantes A, Figer A, Lledo G, Flesch M, Buyse M, et al. OPTIMOX1: a randomized study of FOLFOX4 or FOLFOX7 with oxaliplatin in a stop-and-go fashion in advanced colorectal cancer-a GERCOR study. J Clin Oncol. 2006;24:394-400.

21. Chibaudel B, Maindrault-Goebel F, Lledo G, Mineur L, André T, Bennamoun M, et al. Can chemotherapy be discontinued in unresectable metastatic colorectal cancer? The GERCOR OPTIMOX2 study. J Clin Oncol. 2009;27:5727-33.

22. Adams RA, Meade AM, Seymour MT, Wilson RH, Madi A, Fisher D, et al. Intermittent versus continuous oxaliplatin and fluoropyrimidine combination chemotherapy for first-line treatment of advanced colorectal cancer: results of the randomised phase 3 MRC COIN trial. Lancet Oncol. 2011;12:642-53.

23. Nakayama G, Kodera Y, Yokoyama H, Okuda N, Watanabe T, Tanaka C, et al. Modified FOLFOX6 with oxaliplatin stopand-go strategy and oral S-1 maintenance therapy in advanced colorectal cancer: CCOG-0704 study. Int J Clin Oncol. 2011;16:506-11.

24. Tezuka T, Hamada C, Ishida H, Ooshiro M, Matsuoka H, Kawasaki S, et al. Phase II clinical study of modified FOLFOX7 (intermittent oxaliplatin administration) plus bevacizumab in patients with unresectable metastatic colorectal cancer-CRAFT study. Invest New Drugs. 2013;5:1321-9.

25. Díaz-Rubio E, Gómez-España A, Massutí B, Sastre J, Abad A, Valladares M, et al. First-line XELOX plus bevacizumab followed by XELOX plus bevacizumab or single-agent bevacizumab as maintenance therapy in patients with metastatic colorectal cancer: the phase III MACRO TTD study. Oncologist. 2012;17:15-25.

26. Simkens LH, van Tinteren H, May A, ten Tije AJ, Creemers GJ, Loosveld OJ, et al. Maintenance treatment with capecitabine and bevacizumab in metastatic colorectal cancer (CAIRO3): a phase 3 randomised controlled trial of the Dutch Colorectal Cancer Group. Lancet. 2015;385:1843-52.

27. Nakayama G, Ishigure K, Yokoyama H, Uehara K, Kojima H, Ishiyama A, et al. The efficacy and safety of CapeOX plus bevacizumab therapy followed by capecitabine plus bevacizumab maintenance therapy in patients with metastatic colorectal cancer: a multi-center, single-arm, phase II study (CCOG-0902). BMC Cancer. 2017;17:243.

28. National Cancer Institute. National Cancer Institute Common Toxicity Criteria, version 4.0. https://ctep.cancer.gov/protocolDevelopment/electronic_applications/ctc.htm\#ctc_40. Accessed 06 June 2011.

29. Lersch C, Schmelz R, Eckel F, Erdmann J, Mayr M, Schulte-Frohlinde E, et al. Prevention of oxaliplatin-induced peripheral sensory neuropathy by carbamazepine in patients with advanced colorectal cancer. Clin Colorectal Cancer. 2002;2:54-8. 
30. Levi F, Perpoint B, Garufi C, Focan C, Chollet P, Depres-Brummer P, et al. Oxaliplatin activity against metastatic colorectal cancer. A phase II study of 5-day continuous venous infusion at circadian rhythm modulated rate. Eur $\mathrm{J}$ Cancer. 1993;29A:1280-4.

31. Boku N, Ohtsu A, Hyodo I, Shirao K, Miyata Y, Nakagawa K, et al. Phase II study of oxaliplatin in Japanese patients with metastatic colorectal cancer refractory to fluoropyrimidines. Jpn J Clin Oncol. 2007;37:440-5.

32. Kosugi C, Koda K, Ishibashi K, Yoshimatsu K, Tanaka S, Kato R, et al. Safety of mFOLFOX6/XELOX as adjuvant chemotherapy after curative resection of stage III colon cancer: phase II clinical study (The FACOS study). Int J Colorectal Dis. 2018; 33:809-817.

33. Schmoll HJ, Cunningham D, Sobrero A, Karapetis CS, Rougier P, Koski SL, et al. Cediranib with mFOLFOX6 versus bevacizumab with mFOLFOX6 as first-line treatment for patients with advanced colorectal cancer: a double-blind, randomized phase III study (HORIZON III). J Clin Oncol. 2012;30:3588-95.

34. Hochster HS, Grothey A, Hart L, Rowland K, Ansari R, Alberts S, et al. Improved time to treatment failure with an intermittent oxaliplatin strategy: results of CONcePT. Ann Oncol. 2014;25:1172-8.

35. Yalcin S, Uslu R, Dane F, Yilmaz U, Zengin N, Buyukunal E, et al. Bevacizumab + capecitabine as maintenance therapy after initial bevacizumab + XELOX treatment in previously untreated patients with metastatic colorectal cancer: phase III 'Stop and Go' study results-a Turkish Oncology Group Trial. Oncology. 2013;85:323-35.

\section{Tables}

Table 1 Patient baseline characteristics 


\begin{tabular}{|c|c|c|c|}
\hline \multirow[t]{2}{*}{ Characteristic } & \multicolumn{2}{|c|}{ Enrolled } & \\
\hline & \multicolumn{2}{|l|}{$\mathrm{n}=47(\%)$} & \\
\hline \multicolumn{2}{|l|}{ Age (years) } & \multicolumn{2}{|c|}{67 (range: 46-83) } \\
\hline \multirow[t]{2}{*}{ Sex } & Male & 29 & $(61.7)$ \\
\hline & Female & 18 & $(38.3)$ \\
\hline \multicolumn{4}{|c|}{ ECOG performance status } \\
\hline & 0 & 41 & $(87.2)$ \\
\hline & 1 & 6 & $(12.8)$ \\
\hline \multicolumn{4}{|l|}{ Primary site } \\
\hline & Colon & 26 & $(55.3)$ \\
\hline & Rectum & 21 & $(44.7)$ \\
\hline \multicolumn{4}{|c|}{ Primary tumor resection } \\
\hline & Colon & 21 & $(44.7)$ \\
\hline & Rectum & 14 & $(29.8)$ \\
\hline \multicolumn{4}{|c|}{ Histological type } \\
\hline & tub1 & 9 & (19.1) \\
\hline & tub2 & 37 & (78.7) \\
\hline & por & 1 & $(2.1)$ \\
\hline & muc & 0 & \\
\hline & sig & 0 & \\
\hline & ecc & 0 & \\
\hline \multicolumn{4}{|c|}{ Site of metastasis } \\
\hline & Liver & 31 & $(65.9)$ \\
\hline & Lung & 15 & (31.9) \\
\hline & Lymph node & 16 & $(34.0)$ \\
\hline & Peritoneum & 10 & $(21.3)$ \\
\hline & Other & 10 & $(21.3)$ \\
\hline \multicolumn{4}{|c|}{ Primary adjuvant chemotherapy } \\
\hline & No & 36 & $(76.6)$ \\
\hline & Yes & 11 & $(23.4)$ \\
\hline
\end{tabular}

Abbreviations: ECOG Eastern Cooperative Oncology Group, tub1 well-differentiated tubular adenocarcinoma, tub2 moderately-differentiated tubular adenocarcinoma, por poorly differentiated adenocarcinoma, sig signet-ring cell carcinoma, muc mucinous adenocarcinoma, ecc endocrine cell carcinoma

Table 2 Treatment results 


\begin{tabular}{|c|c|c|}
\hline \multicolumn{3}{|l|}{$(n=47)$} \\
\hline & $\mathrm{n}$ & range or percent \\
\hline \multirow[t]{2}{*}{ Treatment cycles ${ }^{\mathrm{a}}$} & 10 & $(1-15)$ \\
\hline & & (average: 10.2) \\
\hline \multicolumn{3}{|l|}{ Total dose ${ }^{a}$} \\
\hline Oxaliplatin (mg) & 1132.5 & $(150.0-2200.0)$ \\
\hline Oxaliplatin (mg/m²) & 649.1 & $(105.1-1031.5)$ \\
\hline Capecitabine & 19609.9 & $(2100.4-34773.2)$ \\
\hline Bevacizumab & 2649.9 & $(269.5-4487.7)$ \\
\hline \multicolumn{3}{|l|}{ Relative dose intensity ${ }^{a}$} \\
\hline Oxaliplatin & 94 & $(50.0-100)$ \\
\hline Capecitabine & 92.0 & $(58.3-100)$ \\
\hline Bevacizumab & 100.0 & $(78.9-100)$ \\
\hline Continuation of planned cycles ${ }^{a}$ & 22 & $(46.8 \%)$ \\
\hline Study treatment discontinuation ${ }^{a}$ & 25 & $(53.2 \%)$ \\
\hline \multicolumn{3}{|l|}{ Causes of study treatment discontinuation } \\
\hline Progressive disease & 9 & $(19.1 \%)$ \\
\hline Conversion to operation & 3 & $(6.4 \%)$ \\
\hline Adverse event & 8 & $(17.0 \%)$ \\
\hline Allergic reaction & 0 & \\
\hline Peripheral sensory neuropathy & 3 & \\
\hline Anorexia & 1 & \\
\hline General fatigue & 1 & \\
\hline Diarrhea & 1 & \\
\hline Febrile neutropenia & 0 & \\
\hline Thrombocytopenia & 0 & \\
\hline Perforation & 1 & \\
\hline Hand-foot syndrome & 1 & \\
\hline Oral intake not possible due to small bowel obstruction & 1 & $(2.1 \%)$ \\
\hline Patient refusal & 1 & $(2.1 \%)$ \\
\hline Death & 2 & $(4.3 \%)$ \\
\hline a Median (range) & & \\
\hline
\end{tabular}


Table 3 Objective tumor response

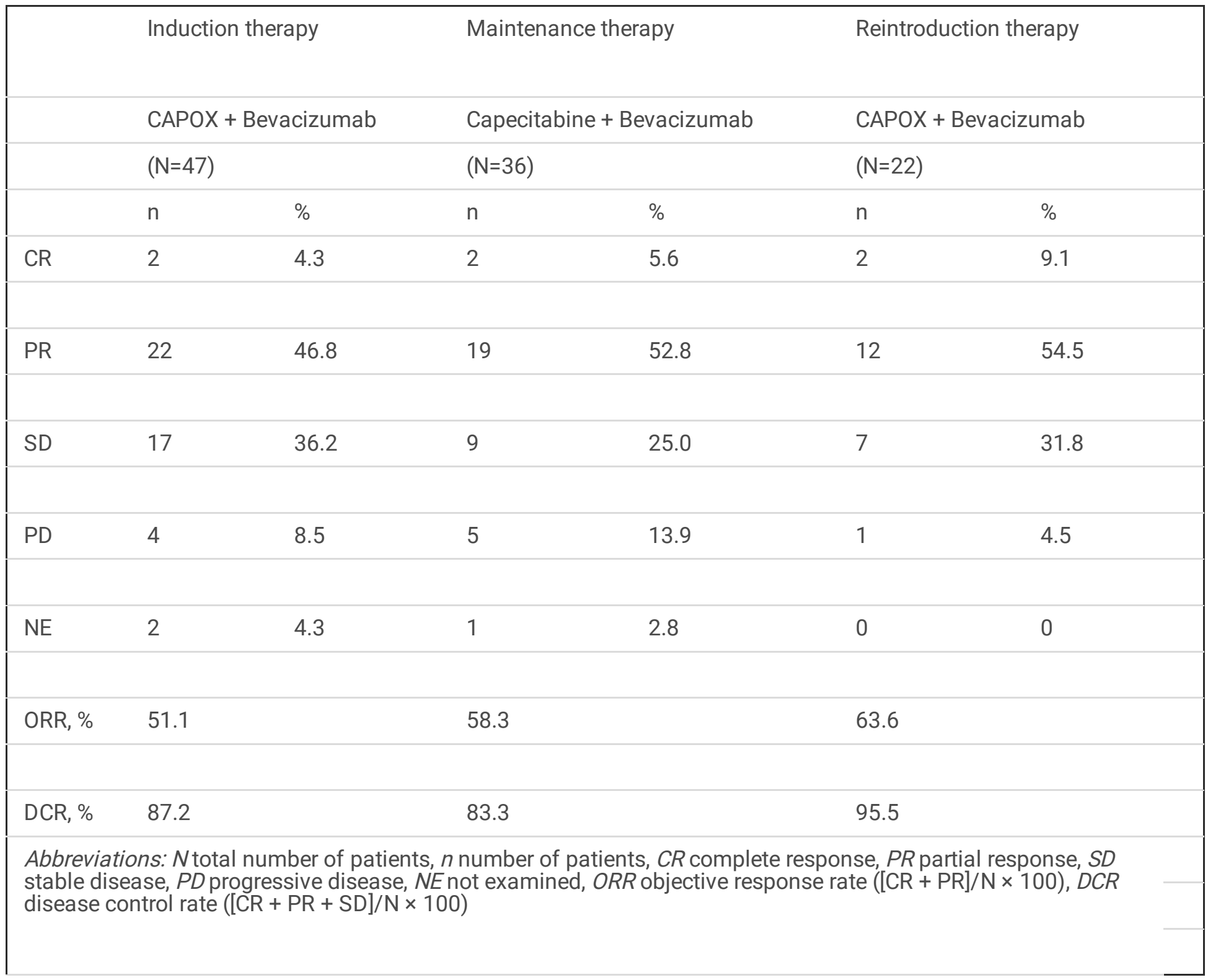

Table 4 Adverse events according to the NCI-CTCAE criteria, version 4.0 


\begin{tabular}{|c|c|c|c|c|c|c|c|c|c|c|c|c|}
\hline & \multicolumn{4}{|c|}{ Induction therapy } & \multicolumn{4}{|c|}{ Maintenance therapy } & \multicolumn{4}{|c|}{ Reintroduction therapy } \\
\hline & \multicolumn{4}{|c|}{ CAPOX + Bevacizumab } & \multicolumn{4}{|c|}{ Capecitabine + Bevacizumab } & \multicolumn{4}{|c|}{ CAPOX + Bevacizumab } \\
\hline & \multicolumn{4}{|c|}{$(\mathrm{N}=47)$} & \multicolumn{4}{|c|}{$(\mathrm{N}=36)$} & \multicolumn{4}{|c|}{$(\mathrm{N}=22)$} \\
\hline & \multicolumn{2}{|c|}{ All grades } & \multicolumn{2}{|c|}{$\begin{array}{l}\text { Grade } \\
\geq 3\end{array}$} & \multicolumn{2}{|c|}{ All grades } & \multicolumn{2}{|c|}{$\begin{array}{l}\text { Grade } \\
\geq 3\end{array}$} & \multicolumn{2}{|c|}{ All grades } & \multicolumn{2}{|c|}{$\begin{array}{l}\text { Grade } \\
\geq 3\end{array}$} \\
\hline & $\mathrm{n}$ & $\%$ & $\mathrm{n}$ & $\%$ & $\mathrm{n}$ & $\%$ & $\mathrm{n}$ & $\%$ & $\mathrm{n}$ & $\%$ & $\mathrm{n}$ & $\%$ \\
\hline \multicolumn{13}{|l|}{ Hematologic toxicities } \\
\hline Leukopenia & 3 & 6.4 & 0 & 0 & 2 & 5.6 & 0 & 0 & 3 & 13.6 & 0 & 0 \\
\hline Neutropenia & 7 & 14.9 & 1 & 2.1 & 5 & 13.9 & 0 & 0 & 2 & 9.1 & 0 & 0 \\
\hline $\begin{array}{l}\text { Febrile } \\
\text { neutropenia }\end{array}$ & 0 & 0 & 0 & 0 & 0 & 0 & 0 & 0 & 0 & 0 & 0 & 0 \\
\hline Anemia & 5 & 10.6 & 0 & 0 & 2 & 5.6 & 1 & 2.8 & 0 & 0 & 0 & 0 \\
\hline Thrombocytopenia & 2 & 4.3 & 0 & 0 & 2 & 5.6 & 0 & 0 & 0 & 0 & 0 & 0 \\
\hline ALT & 1 & 2.1 & 0 & 0 & 1 & 2.8 & 0 & 0 & 1 & 4.5 & 0 & 0 \\
\hline AST & 1 & 2.1 & 0 & 0 & 0 & 0 & 0 & 0 & 1 & 4.5 & 0 & 0 \\
\hline T.Bil & 2 & 4.3 & 0 & 0 & 2 & 5.6 & 0 & 0 & 0 & 0 & 0 & 0 \\
\hline Creatinine & 0 & 0 & 0 & 0 & 0 & 0 & 0 & 0 & 0 & 0 & 0 & 0 \\
\hline \multicolumn{13}{|l|}{$\begin{array}{l}\text { Non-hematologic } \\
\text { toxicities }\end{array}$} \\
\hline General fatigue & 15 & 31.9 & 0 & 0 & 9 & 25.0 & 0 & 0 & 7 & 31.8 & 0 & 0 \\
\hline Stomatitis & 9 & 19.1 & 0 & 0 & 6 & 16.7 & 0 & 0 & 4 & 18.2 & 1 & 4.5 \\
\hline Nausea/Vomiting & 9 & 19.1 & 0 & 0 & 4 & 11.1 & 0 & 0 & 4 & 18.2 & 0 & 0 \\
\hline Appetite loss & 18 & 38.3 & 1 & 2.1 & 5 & 13.9 & 0 & 0 & 2 & 9.1 & 0 & 0 \\
\hline Diarrhea & 10 & 21.3 & 1 & 2.1 & 2 & 5.6 & 0 & 0 & 2 & 9.1 & 0 & 0 \\
\hline $\begin{array}{l}\text { Peripheral sensory } \\
\text { neuropathy }\end{array}$ & 26 & 55.3 & 1 & 2.1 & 16 & 44.4 & 1 & 2.8 & 13 & 59.1 & 0 & 0 \\
\hline Allergic reaction & 2 & 4.3 & 1 & 2.1 & 0 & 0 & 0 & 0 & 0 & 0 & 0 & 0 \\
\hline $\begin{array}{l}\text { Hand-foot } \\
\text { syndrome }\end{array}$ & 18 & 38.3 & 0 & 0 & 18 & 50.0 & 1 & 2.8 & 12 & 54.5 & 0 & 0 \\
\hline \multicolumn{13}{|l|}{$\begin{array}{l}\text { Bevacizumab-related } \\
\text { toxicities }\end{array}$} \\
\hline Hypertension & 8 & 17.0 & 2 & 4.3 & 6 & 16.7 & 2 & 5.6 & 5 & 22.7 & 1 & 4.5 \\
\hline Proteinuria & 8 & 17.0 & 0 & 0 & 6 & 16.7 & 0 & 0 & 5 & 22.7 & 0 & 0 \\
\hline Bleeding & 1 & 2.1 & 0 & 0 & 3 & 8.3 & 0 & 0 & 2 & 9.1 & 0 & 0 \\
\hline $\begin{array}{l}\text { Digestive tract } \\
\text { perforation }\end{array}$ & 1 & 2.1 & 0 & 0 & 0 & 0 & 0 & 0 & 0 & 0 & 0 & 0 \\
\hline Thrombosis & 1 & 2.1 & 1 & 2.1 & 0 & 0 & 0 & 0 & 0 & 0 & 0 & 0 \\
\hline
\end{tabular}




\section{Figures}

Figure 1

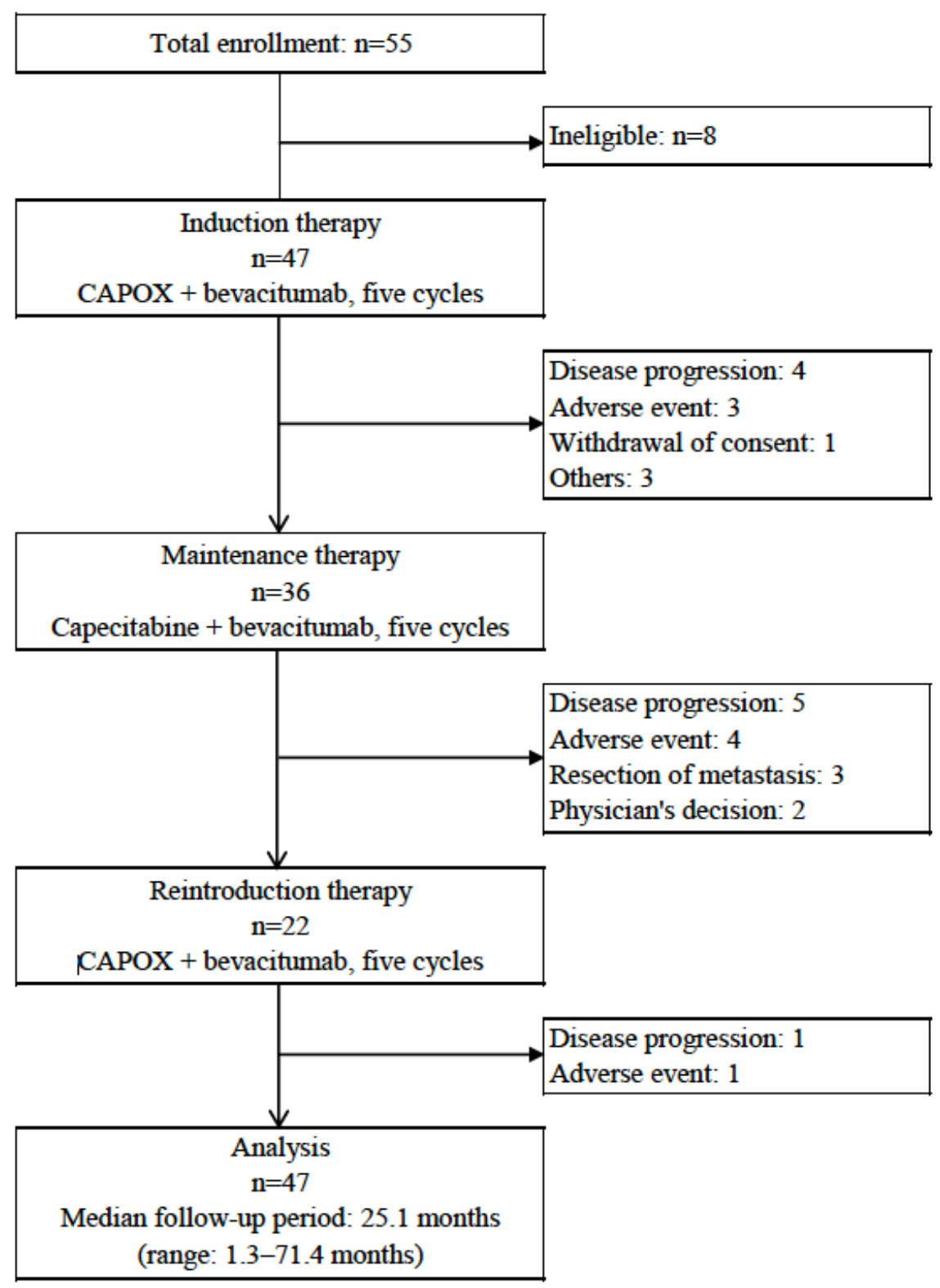

Figure 1

Flowchart of the study participants Forty-seven patients enrolled between March 2011 and August 2013 received the initial CAPOX plus bevacizumab therapy. The maintenance therapy with capecitabine and bevacizumab was introduced in 36 patients, and oxaliplatin was reintroduced in 22 patients. Abbreviations: CAPOX capecitabine plus oxaliplatin 
Figure 2

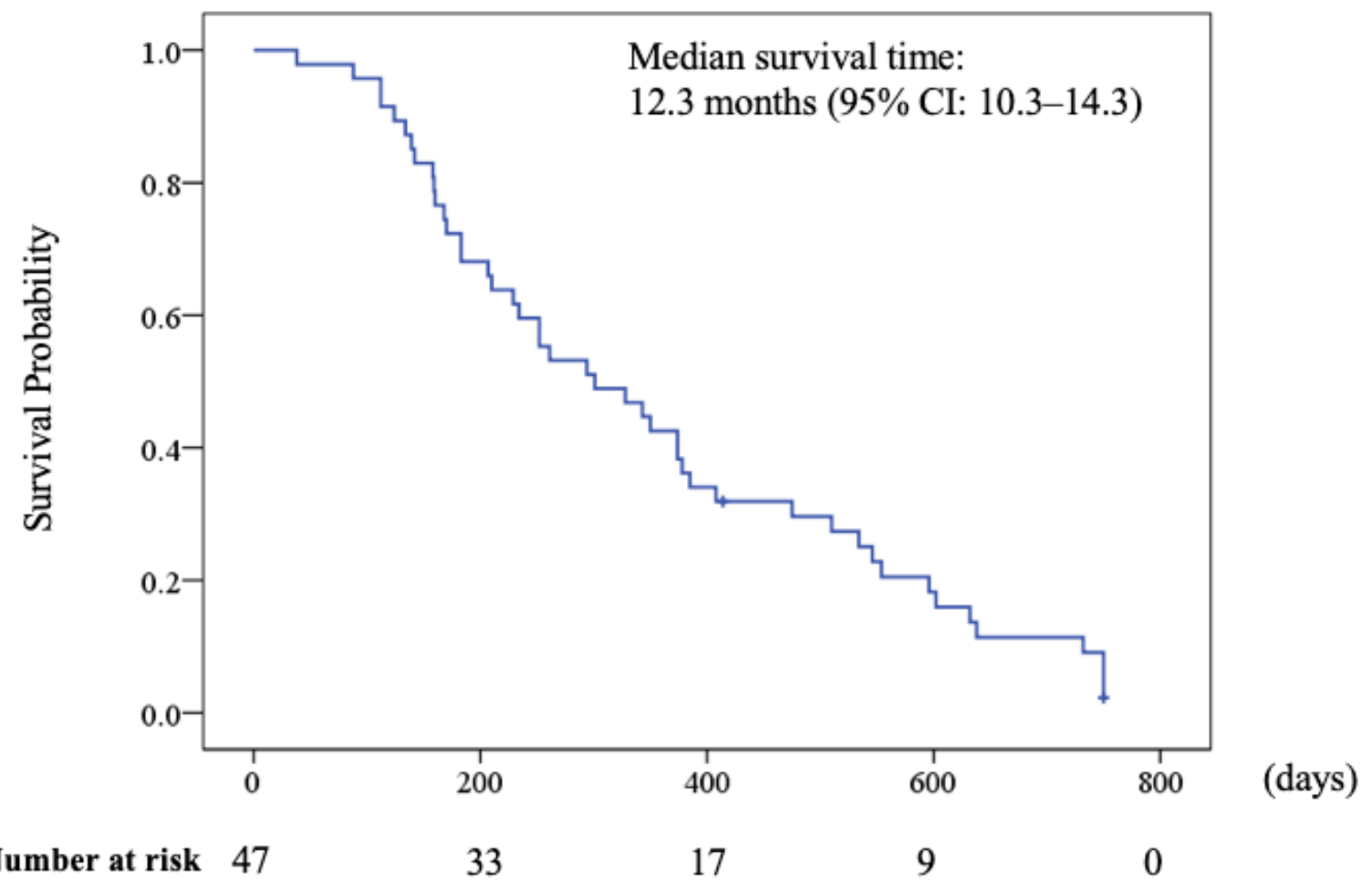

Figure 2

Kaplan-Meier estimate of time to treatment failure Median time to treatment failure was 12.3 months $(95 \%$ Cl, $10.3-$ 14.3). Abbreviations: $\mathrm{Cl}$ confidence interval 
Figure 3

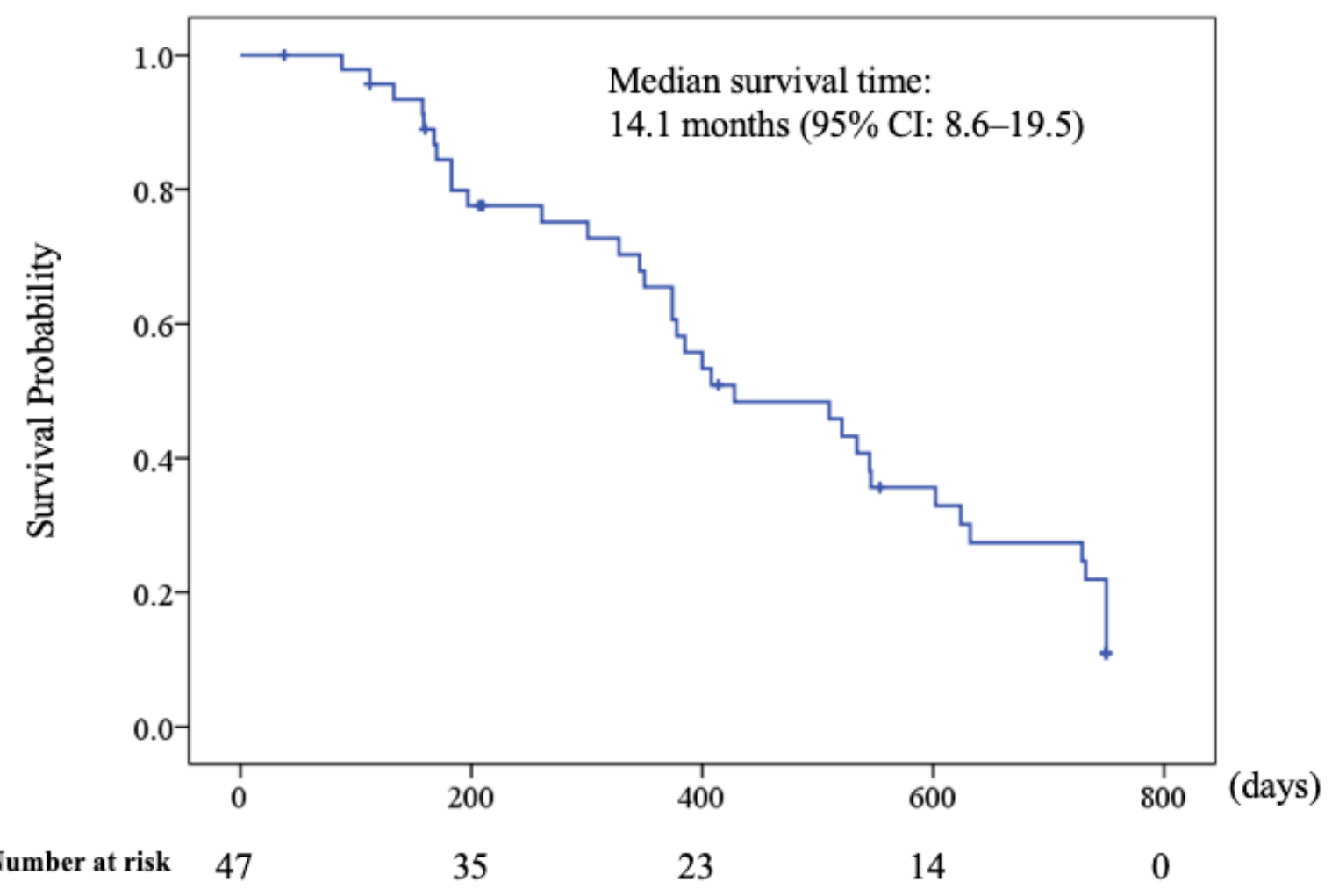

Figure 3

Kaplan-Meier estimate of progression-free survival Median progression-free survival was 14.1 months (95\% Cl: 8.619.5). Abbreviations: $\mathrm{Cl}$ confidence interval 